\title{
Consultores Ad hoc - 2017
}

Para atender à demanda de avaliação dos artigos submetidos ao BOLEMA temos contado com os membros do Conselho Editorial ${ }^{1}$ e com colaboradores ad hoc. Durante o ano de 2017, colaboraram com o BOLEMA, como pareceristas ad hoc, os seguintes pesquisadores:

Adlai Ralph Detoni (UFJF-MG), Adriana Richit (UFFS-RS), Alessandro Jaques Ribeiro (UFABC-SP), Ana Leticia Losano (UNICAMP-SP), Angela Marta Pereira das Dores Savioli (UEL-PR), Angélica Vega (Universidad de Chile - Chile), Ângela Freira (UFBA-BA), Arlete de Jesus Brito (UNESP-SP), Astrid Morales ((Pontificia Universidad Catolica de Valparaiso Chile), Carlos Henrique Gonçalves (USP-SP), Carmen Batanero (Universidad de Granada Espanha), Cesar Donizetti Pereira Leite (UNESP-SP), Cristina Esteley (Universidad Nacional de Cordoba - Argentina), Daniel Reinholz (San Diego State University - EUA), Dario Fiorentini (UNICAMP-SP), Denizalde Pereira (UNEMAT-MT), Dinazar Escudero-Avila (Benemerita Universidad Autonoma de Puebla - México), Edna Maura Zuffi (USP-SP), Eugenio Lizarde (Escuela Normal Rural Gral. Matías Ramos Santos - México), Fabiane Cristina Höpner Noguti (UFSM-RS), Ferdinand Rivera (San Jose State University - EUA), Gerson Pastre de Oliveira (PUC-SP), Gilda Lisboa Guimarães (UFPE-PE), Gilberto Vieira (Faculdade de Tecnologia de Sao Jose dos Campos - SP), Helena Rocha (Universidade Nova de Lisboa - Portugal), Irene Cazorla (UESC-BA), Jean-Claude Régnier (Université de Lyon França), Jhony Alexander Villa-Ochoa (Universidad de Antioquia - Colombia), Jose Carlos Cifuentes Vasquez (UFPR-PR), José Carlos Leivas (UNIFRA-RS), José Armando Valente (UNICAMP-SP), José Luiz Freitas (UFMS-MS), Juan Godino (Universidad de Granada Espanha), Jussara de Loiola Araújo (UFMG-MG), Kate O'Hara (Cleveland State University College - EUA), Keith Weber(Rutgers The State University of New Jersey - EUA), Lourdes de la Rosa Onuchic (UNESP-SP), Lourdes Maria Werle Almeida (UEL-PR), Luana Oliveira Sampaio (UFSB-BA), Luis Roberto Pino-Fan (Universidad de Los Lagos - Chile), Luz Valoyes (Universidad de Chile - Chile), Mani Arguelles (Columbia High School - EUA), Manoel Oriosvaldo Moura (USP-SP), Maria de Fátima Teixeira Barreto (UFG-GO), Maria Celeste Reis Fernandes de Souza (Universidade Vale do Rio Doce - MG), Marilena Bittar (UFMS-MS), María Soledad Estrella (Pontificia Universidad Catolica de Valparaiso - Chile), Miguel Montes (Universidad de Huelva - Espanha), Miguel Rodríguez Wilhelmi (Universidad Publica de Navarra - Espanha), Nelson Pirola (UNESP-SP), Nélia Amado (Universidade do Algarve - Portugal), Orlando Figueiredo (UNESP-SP), Paola Valero (Universidad de Aalborg - Dinamarca), Paul Dawkins (Northern Illinois University - EUA), Rosilda dos Santos Morais (UNIFESP-SP), Rute Borba (UFPE-PE), Silvana Santos (UFV-MG), Silvana Matucheski (IFPR-PR), Sérgio Carrazedo Dantas (UNESPAR-PR), Tiago Kluber (UNIOESTE-PR), Verônica Gitirana Gomes Ferreira (UFPE-PE), Vicenc Font (Universitat de Barcelona Espanha), Victor Giraldo (UFRJ-RJ), Wilson Gordillo (Universidad Distrital Francisco José de Caldas - Colombia).

\footnotetext{
${ }^{1}$ Os membros do Conselho Editorial estão disponíveis em: http://www.scielo.br/revistas/bolema/pedboard.htm.
} 